\title{
Carnap on Theoretical Terms: Structuralism without Metaphysics
}

\author{
Michael Friedman \\ Department of Philosophy \\ Stanford University \\ Stanford, CA 94305 \\ Email: mlfriedman@stanford.edu
}

\begin{abstract}
Both realists and instrumentalists have found it difficult to understand (much less accept) Carnap's developed view on theoretical terms, which attempts to stake out a neutral position between realism and instrumentalism. I argue that Carnap's mature conception of a scientific theory as the conjunction of its Ramsey sentence and Carnap sentence can indeed achieve this neutral position. To see this, however, we need to see why the Newman problem raised in the context of recent work on structural realism is no problem for Carnap's conception; and we also need to locate Carnap's work on theoretical terms within his wider program of Wissenschaftslogik or the logic of science.
\end{abstract}

Carnap's distinctive approach to theoretical terms dates from beginnings of his semantic period in the years following the publication of The Logical Syntax of Language (1934). Particularly important, in this context, is his monograph Foundations of Logic and Mathematics (1939), where the partial interpretation view of theoretical terms first emerges. This view is further articulated in "The Methodological Character of Theoretical Concepts" (1956), and it is then connected with Carnap's use of the Ramsey sentence in "Beobachtungssprache und Theoretische Sprache" (1958)—through the mediation, as Stathis Psillos first documented, of Hempel's "The Theoretician's Dilemma" (1958). ${ }^{\mathrm{i}}$ Carnap continued to work on the Ramsey sentence representation of theories (and the closely related representation using Hilbert's $\varepsilon$-operator) throughout the 
late 1950s and early 1960s, culminating in Philosophical Foundations of Physics in 1966 (based on a typescript of his Fall 1958 seminar at UCLA made by Martin Gardiner).

Carnap makes it very clear, beginning in the "Methodological Character" essay, that he intends his approach to dissolve rather than solve the "ontological" dispute between realism and instrumentalism. In line with his general approach to ontological disputes developed in "Empiricism, Semantics, and Ontology" (1950), Carnap regards this dispute—conceived as theoretical—as involving a philosophical "pseudo-question" about the "reality" (in an objectionably metaphysical sense) of a certain general kind of entities (in this case theoretical entities), and his strategy (as is typical with him) is rather to argue that both realism and instrumentalism, in so far as they are not entangled with philosophical pseudo-problems, have an important kernel of truth; where they purport to disagree with one another, however, we leave the firm ground of scientific truth in favor of the confused "inextricable tangle" of traditional philosophy.

Carnap's definitive formulation of what Psillos (1999, chapter 3) has very helpfully called his "neutralism" comes at the very end of the chapter on the Ramsey sentence in the second (1974) edition of Philosophical Foundations of Physics (crucially revised with respect to this very issue shortly before his death in 1970):

It is obvious that there is a difference between the meanings of the instrumentalist and the realist ways of speaking. My own view, which I shall not elaborate here, is essentially this. I believe that the question should not be discussed in the form: "Are theoretical entities real?" but rather in the form: "Shall we prefer a language of physics (and of science in general) that contains theoretical terms, or a language 
without such terms?" From this point of view the question becomes one of preference and practical decision. ${ }^{4}(1974$, p. 256)

And the footnote to the last sentence explicitly refers the reader to "Empiricism, Semantics, and Ontology"-which is not cited in either the first edition in 1966 or in the "Methodological Character" essay in $1958 .{ }^{\mathrm{ii}}$

Carnap's attempt at neutrality has not satisfied either instrumentalists or realists. The leading defender of instrumentalism of our time, Bas van Fraassen, regards logical positivism as perhaps the most important earlier twentieth-century implementation of the empiricist approach he favors; and van Fraassen regards Carnap's "Methodological Character" paper as "in many ways, the culmination of the positivist programme" (1980, 13). Nevertheless, van Fraassen is also very clear and explicit that Carnap's particular implementation, based on formal logic and a fundamentally linguistic or "syntactic" view of theories, is ultimately hopeless:

Perhaps the worst consequence of the syntactic approach was the way it focussed attention on philosophically irrelevant technical questions. It is hard not to conclude that those discussions of axiomatizability in restricted vocabularies, 'theoretical terms', Craig's theorem, 'reduction sentences', 'empirical languages', Ramsey and Carnap sentences, were one and all off the mark—-solutions to purely self-generated problems, and philosophically irrelevant. $(1980,56)$

Thus, despite the fact that Carnap's understanding of the Ramsey sentence leads to a conception of the empirical content of scientific theories which (as we shall see) is 
virtually identical with van Fraassen's own proposal for what an "agnostic" instrumentalist should assert, van Fraassen has very little patience for Carnap's general approach.

It is striking, by contrast, that Stathis Psillos, who has now mounted the most detailed and insightful defense of scientific realism in the wake of van Fraassen's work (Psillos, 1999), devotes an entire chapter to the topic of "Carnap's neutralism." He not only has very considerable patience for Carnap's approach, but, in the course of his work, Psillos has also made important contributions to Carnap scholarship (compare note i above). With respect to the realism-instrumentalism debate, in particular, Psillos sees important connections between Carnap's use of the Ramsey sentence and contemporary structural realism. In the end, however, Psillos judges that Carnap's neutralism failsdue to a general problem for the Ramsey sentence approach first raised by M. H. A. Newman (1928) in connection with Bertrand Russell's early version of structural realism. ${ }^{\text {iii }}$ The problem, roughly, is that, if the Ramsey sentence is empirically adequate (if all its observational consequences are true), then it is necessarily true as well-true as a simple matter of (higher-order) logic. So it does not seem, after all, that the Ramsey sentence, as Carnap proposes, can faithfully represent the synthetic content that our original theory is supposed to have.

As Psillos himself puts the point $(1999,62)$ : "In the end, if no constraints are imposed on the range of the variables of the Ramsey sentence, it is a trivial and a priori assertion that there are electrons, etc. And this is clearly absurd. For, to say the least, it appears obvious that the [original, un-Ramsified] theory . . could be false, even though it is empirically adequate." Indeed, as Psillos explains several pages later, he takes the 
Newman problem to undermine all forms of structural realism, in so far as it shows that some or another kind of "non-structural information" (about which properties and relations are "natural" or the like) must therefore be added on pain of incoherence.

I suggested above that, despite his lack of patience with Carnap's approach, van Fraassen's view of what a scientific theory should properly assert is virtually identical with Carnap's conception of the Ramsey sentence representation of a theory's synthetic or empirical content. ${ }^{\text {iv }}$ Van Fraassen thinks that we should only assert that the observational phenomena are embeddable into an abstract model for the theory, and the Ramsey sentence, on Carnap's account, asserts precisely the same-that there is some abstract (mathematical) model of the theory such that all observable phenomena behave in the way that the theory requires $(1966 / 74,254-5)$ : "Some physicists are content to think about such terms as 'electron' in the Ramsey way. They evade the question of existence by stating that there are certain observable events, in bubble chambers and so on, that can be described by certain mathematical functions, within the framework of a certain theoretical system. Beyond that they will assert nothing."

Nevertheless, it is equally true, as Psillos has argued, that Carnap's view also has much in common with contemporary structural realism. Indeed, Carnap himself ends Philosophical Foundations of Physics with what looks like a ringing endorsement of that view:

Some physicists believe that there is a good chance for a new breakthrough [in our understanding of quantum mechanics] in the near future. Whether it will be soon or later, we may trust-provided the world's leading statesman refrain from the ultimate folly of nuclear 
war and permit humanity to survive-that science will continue to make great progress and lead us to ever deeper insights into the structure of the world. (1966/74, 291-292)

The circumstance that Carnap has this much in common with both van Fraassen's instrumentalism and contemporary structural realism suggests, at least to me, that Carnap's attempt at neutrality may have succeeded after all. He may in fact have articulated a version of structuralism that recognizes the strengths of both instrumentalism and realism while simultaneously avoiding the philosophical "pseudoquestions" on which they appear substantively to differ. But this can only be fully appreciated, I shall argue, when we place Carnap's views on theoretical terms within his wider conception of the task of philosophy of science-which he calls Wissenschaftslogik, the "logic of science"-more generally.

Carnap's serious discussion of what is now called the problem of theoretical terms begins, as I have suggested, with Foundations of Logic and Mathematics in 1939. There, however, he does not in fact use the concept of a "theoretical" term, nor does he engage with the debate between realism and instrumentalism. Carnap instead distinguishes between "elementary" and "abstract" terms-where the latter are introduced as terms that occur towards the end of a more-or-less continuous series beginning with very simple ordinary language predicates ("elementary" terms) of observable things ('bright', 'dark', 'red', 'blue') and ending with the most abstruse terms of contemporary mathematical physics ('electric field', 'electric potential', 'wave function'). We can, Carnap says, state standard semantical rules (such as 'the term 'te' designates temperature') for any of these terms. "But," he continues (\$24, 204), 
"suppose we have in mind the following purpose for our syntactical and semantical description of the system of physics: the description of the system shall teach a layman to understand it, i.e., to enable him to apply it to his observations in order to arrive at explanations and predictions." We cannot presuppose that the layman already understands the more abstract terms in question, so we cannot introduce them in the semantical metalanguage via standard rules of designation. The alternative is to give no direct semantic interpretation at all for the abstract terms; view them as having only implicit definitions within the total language of physics; and then use the semantic interpretations (rules of designation) we can legitimately give for the more concrete or elementary terms to anchor the whole system on "the solid ground of observable facts" $(\$ 24,207)$.

Carnap calls this kind of interpretation of the abstract terms an "indirect interpretation"-which, as he points out, is, in an important sense, "incomplete" (ibid.); and this is clearly the same idea as what he will later, in the "Methodological Character" essay, call a partial interpretation (of the theoretical terms). In 1939, however, Carnap is primarily addressing a problem about meaning or understanding (rather than an "ontological" problem); and he makes it very clear, in particular, that this perspective on "abstract" (theoretical) terms takes its starting point from the increasing use of the abstract (Hilbertian) axiomatic method in modern mathematical physics:

The development of physics in recent centuries, and especially in the past few decades, has more and more led to that method in the construction, testing, and application of physical theories which we call formalization, i.e., the construction of a calculus supplemented by 
a [partial or incomplete-MF] interpretation. It was the progress of knowledge and the particular structure of the subject matter that suggested and made practically possible this increasing formalization. In consequence it became more and more possible to forego an "intuitive understanding" of the abstract terms and axioms and theorems formulated with their help. $(\S 25,209)$

Carnap sees the theories of relativity and quantum mechanics as the culmination of this development-where the use of highly abstract terms introduced by something like Hilbertian implicit definitions (terms such as 'electron', 'electromagnetic field', 'metrictensor', and 'psi-function') has become a pervasive and essential feature of physical practice. $^{v}$

According to Carnap's account in the "Methodological Character" essay, only the observational terms of a scientific theory are semantically interpreted (by specifying observable properties and relations as their designata). The theoretical terms, by contrast, are semantically uninterpreted, and are only implicitly defined, in the sense of Hilbert, by the axioms and postulates of the relevant theory (e.g., Maxwell's equations for the electromagnetic field). Among these axioms and postulates, however, are mixed sentences or correspondence rules, which set up (lawlike) relationships among theoretical and observational terms; and, in this way, the theoretical terms and sentences receive a partial interpretation in terms of the connections they induce among observables. For example, Maxwell's equations, in the presence of suitable correspondence rules relating values of the electromagnetic field to actual measurements 
(of electric and magnetic intensities, and the like), generate observable predictions and thus have empirical content.

Are we thereby "ontologically committed" to the existence of a mysterious unobservable entity corresponding to our term for the electromagnetic field? Here, unlike in 1939, Carnap explicitly considers this question and devotes considerable effort towards trying to defuse it. He stipulates, first of all, that the values of the variables of his theoretical language $L_{T}$ range over a domain of entities including a denumerable sequence isomorphic to the natural numbers and closed over the formation of relations and classes. The domain therefore contains natural numbers, real numbers, sets of real numbers, and so on. "Now," Carnap $(1956,43)$ continues, "we proceed to physics." We conceive space-time points as quadruples of real numbers which thereby belong to the (purely mathematical) domain $D$ we have already constructed. Moreover, physical magnitudes (such as the electromagnetic field) are functions whose arguments are spacetime points and values are real numbers or n-tuples of real numbers. Thus, all the entities needed for values of our variables have already been constructed within our purely mathematical domain $D$. And the same holds, Carnap adds, for the entities of biology, psychology, and the social sciences.

Carnap then cautions the reader:

We have considered some of the kinds of entities referred to in mathematics, physics, psychology, and the social sciences and have indicated that they belong to the [purely mathematical] domain $D$. However, I wish to emphasize here that this talk about the admission of this or that kind of entity as values of variables in $L_{T}$ is only a way 
of speaking intended to make the use of $L_{T}$, and especially the use of quantified variables in $L_{T}$, more easily understandable. Therefore the explanations just given must not be understood as implying that those who accept and use a language are thereby committed to certain "ontological" doctrines in the traditional metaphysical sense. The usual ontological questions about the "reality" (in an alleged metaphysical sense) of numbers, classes, space-time points, bodies, minds, etc., are pseudo-questions without cognitive content. (1956, 44-45)

By contrast, questions about the reality of entities as asked and answered within science — a question, for example, about the reality of the electromagnetic field — can be given a "good scientific meaning" (1956, 45) if, for example, "we understand the acceptance of the reality of the electromagnetic field in the classical sense as the acceptance of a language $L_{T}$ and in it a term, say ' $E$ ', and a set of postulates $T$ which includes the classical laws of the electromagnetic field (say, the Maxwell equations) as postulates for ' $E$ '. For an observer $X$ to 'accept' the postulates of $T$, means here not simply to take $T$ as an uninterpreted calculus, but to use $T$ together with specified correspondence rules $C$ for guiding his expectations by deriving predictions about future observable events from observed events with the help of $T$ and $C . "$

The circumstance that Carnap here proposes a purely mathematical interpretation of the values of the theoretical variables indicates that he is already envisioning a kind of structuralist view-which, as noted above, has much in common with his later use of the Ramsey sentence. However, Carnap does not appeal to the Ramsey sentence here (in 
1956), and, as noted above, he only comes to appreciate the relationship between his developing view of theoretical terms and Ramsey's work after reading Hempel's “Theoretician's Dilemma" in 1958 (see again note i above).

The main point of Hempel's paper is to consider two methods for avoiding or eliminating theoretical terms: re-axiomatization based on Craig's theorem and the Ramsey sentence. The main problem with the first method, according to Hempel, is that it fails to capture the inductive systematization of the observational data effected by theories with theoretical terms. In particular, an object may be observed to exhibit observable behavior which then gives inductive (but not deductive) support for the satisfaction of some theoretical predicate (as, e.g., we can inductively infer that a given object is magnetized from the observable behavior of attracted iron filings). We are then in a position, via the theory, deductively to infer that some other observable behavior will follow (e.g., the motion of a magnetized object may then induce a current in a wire); and, as a result, we have now established an inductive connection between the first observable behavior and the second—one which could certainly not be established in any version of the theory (such as the Craig re-axiomatization) that dispenses with theoretical structure entirely. ${ }^{\mathrm{vi}}$

The Ramsey sentence, Hempel suggests, does not suffer from this defect (or at least not to the same degree), because it retains logical variables referring to the original theoretical entities in place of the original (constant) theoretical terms. The problem now, however, is that, for precisely this reason, the Ramsey sentence continues to have the very same existential commitments as the original theory $(1958,81)$ : “[T]he Ramseysentence associated with an interpreted theory $\mathrm{T}^{\prime}$ avoids reference to hypothetical entities 
only in letter ... rather than in spirit. For it still asserts the existence of certain entities of the kind postulated by $\mathrm{T}^{\prime}$, without guaranteeing any more than does $\mathrm{T}^{\prime}$ that those entities are observable or at least fully characterizable in terms of observables. Hence, Ramseysentences provide no satisfactory way of avoiding theoretical concepts."

Carnap's reply to Hempel in the Carnap Schilpp volume (published in 1963, but likely written in the late 1950s) is especially interesting. For, in the first place, Carnap warmly refers to Hempel (1958) as providing "a thorough and illuminating investigation of the many logical and methodological questions connected with theoretical concepts" (1963, 962), and Carnap here singles out Hempel's distinction between inductive and deductive systematization (ibid.): Hempel explains that, whereas "the $O$-content of a sentence $S$ [the set of observational sentences implied by $S]$. . may serve in certain respects as a substitute for $S$, namely as far as deductive relations among the sentences of [the extended observation language] are concerned, ... the same does not hold for the equally important inductive relations, and . . therefore the concept of $O$-content does not furnish a suitable method for dispensing with theoretical terms. In this view I agree with Hempel.” Indeed, Carnap's remarks in 1956 about how correspondence rules $C$ generate a partial interpretation of an abstract theory $T$ (e.g., Maxwell's equations for the electromagnetic field) furnish a perfect example of this. When we "derive" predictions about future observable events from (prior) observed events we establish precisely an inductive relation between observables in Hempel's sense (as in the example of the magnetized object).

Moreover, in the second place, Carnap also replies to Hempel's (1958) critical remarks on the existential commitments of the Ramsey sentence: 
I agree with Hempel that the Ramsey-sentence does indeed refer to theoretical entities by the use of abstract variables. However, it should be noted that these entities are not unobservable physical objects like atoms, electrons, etc., but rather (at least in the form of the language which I have chosen in [Carnap 1956)]) purely logical-mathematical entities, e.g., natural numbers, classes of such, classes of classes, etc. Nevertheless [the Ramsey sentence of $T$ ] is obviously a factual sentence. It says that the observable events in the world are such that there are numbers, classes of such, etc., which are correlated with the events in a prescribed way and which have among themselves certain relations; and this assertion is clearly a factual statement about the world. $(1963,963)$

Thus, two points are now clear. First, Carnap takes the Ramsey sentence (unlike the Craig re-axiomatization) to provide not only a deductive but also an inductive systematization of the observable phenomena (concerned with both deductive and inductive "relations" between "observable events"). Second, Carnap takes the Ramsey sentence to have synthetic or factual content simply because it thereby constrains the observable phenomena in definite ways. Carnap is not supposing, therefore, that an abstract theory have any synthetic or factual content beyond its empirical adequacy (both deductive and inductive), and, in this respect, he (as I have suggested) is in agreement with van Fraassen but not with contemporary scientific realists like Psillos. The Newman problem is no objection to Carnap. ${ }^{\text {vii }}$ 
This should not be surprising if we keep firmly in mind the fact that theoretical terms, for Carnap, are semantically uninterpreted: we assign no designata to them in our semantical meta-language, and so Tarskian semantics (as Carnap understands it) literally assigns no truth-values at all to purely theoretical sentences. Nevertheless, theoretical terms (as Carnap understands them) still have a crucially important kind of methodological meaning. They establish very significant inductive connections between (fully interpreted) observational sentences-connections that can by no means be recovered in a formulation (like the Craig re-axiomatization) that simply dispenses with theoretical structure entirely-and it is precisely this, in the end, that is secured by a partial interpretation of the theoretical terms via correspondence rules.

It is important to emphasize, in this connection, that Carnap does not propose to replace the original theory $T$ containing constant theoretical terms with a Ramsey sentence replacing these terms with existentially quantified variables. Carnap rather proposes to view the original theory $T$ as the conjunction of the Ramsey sentence and the Carnap sentence, where the latter is the conditional with the Ramsey sentence as antecedent and $T$ itself as consequence. (The conjunction of the Carnap sentence with the Ramsey sentence, is therefore logically equivalent to $T$, and the Carnap sentence gives us a particular existential instantiation, in terms of the original constant theoretical terms of $T$, of the existentially generalized Ramsey sentence.) Thus Carnap says, immediately after his reply to Hempel on the existential commitments of the Ramsey sentence (1963, 963): "I do not propose to abandon the theoretical terms and postulates, as Ramsey suggests, but rather to preserve them in $L_{T}$ and simultaneously to give an important function to the Ramsey-sentences in [the extended observation language]. Their function 
is to serve in the explication of experiential import and, more importantly, in the explication of analyticity." And the way Carnap now explicates analyticity, of course, is by viewing the Ramsey sentence as representing the synthetic part of $T$ while the Carnap sentence represents its analytic part.

I shall return to the question of analyticity in a moment. But I first want to observe that Carnap's preference for his reformulation of $T$ over the Ramsey sentence of $T$ corresponds to his own way of understanding the choice between "the instrumentalist and the realist ways of speaking." For recall that, at the end of the second edition of the chapter on the Ramsey sentence in Philosophical Foundations of Physics, Carnap states that the decision between realism and instrumentalism should be discussed in the form (1974, 256): "Shall we prefer a language of physics (and of science in general) that contains theoretical terms, or a language without such terms?" Carnap's preference, as very clearly stated in the reply to Hempel, is to adopt the former alternative, and so his choice, as he now understands the issue, is to adopt precisely the language of realism.

Does this mean that Carnap is now committed to a realist epistemology and metaphysics (of the kind defended by Psillos, for example), which aims to "explain" the success of science by appealing to pre-existing objective natural kinds in the world, a theory of "factual reference" linking theoretical terms to such objective natural kinds, and an epistemological defense of the "no miracles" argument against the "pessimistic metainduction"? Not at all. Carnap's whole point is to replace the question "are theoretical entities real?" with the question which form of language we should prefer-and prefer for purely pragmatic or practical rather than theoretical reasons. 
But what then are these reasons? Why is Carnap dissatisfied with the Ramsey sentence itself as a formulation of our scientific theory? Suppose we were to attempt directly to make deductions from the Ramsey sentence of the axiomatic theory $T$. We would need to proceed by an instantiation of all the existentially quantified variables, followed by ordinary logico-mathematical reasoning on the basis of this existential instantiation, and concluding with an existential generalization whereby all the existential quantifiers are then reintroduced at the end. This procedure is very complex and cumbersome, and, most importantly, it does not correspond to the way in which we in fact make deductions from axioms in scientific practice-where, in effect, we treat the axioms of $T$ as an Hilbertian implicit definition of the constant theoretical terms of $T$, and we then proceed to engage in ordinary logico-mathematical reasoning from these axioms without worrying about existential instantiation and generalization. ${ }^{\text {viii }}$ It is much more convenient, then, to add the Carnap sentence to the Ramsey sentence-where the Carnap sentence takes over the role of existential instantiation from the Ramsey sentence, and thereby allows us to proceed with ordinary mathematical reasoning in the style of Hilbert without worrying about cumbersome restrictions on existential variables in natural deduction. Whereas existential instantiation, of course, is not a logically valid inference, the Carnap sentence, taken as a non-logical axiom of $T$, is now seen, nonetheless, as an analytic postulate (a meaning postulate) - a conventional choice of (constant) names arbitrarily given to a sequence of values of the existential variables which, by the Ramsey sentence, must (synthetically) exist.

We must therefore modify our earlier claim (arising on Carnap's original version of the partial interpretation conception) that theoretical sentences are assigned no truth 
values. While it is still the case that no semantical rules of designation in the usual sense (of the form 'the term 'te' designates temperature') are provided for theoretical constants, they are now (arbitrarily) assigned a sequence of semantical values that make the theory come out true from among the values ranged over by the theoretical variables (certain sets, functions, and so on). ${ }^{\text {ix }}$ If the Ramsey sentence is true then such a sequence must (synthetically) exist; and, as we have seen, the Ramsey sentence is true just in case all its observational consequences are true. There can therefore be no gap, in Carnap's conception, between the empirical adequacy of a partially interpreted theory and the full (semantical) truth of this same theory.

In this way, in particular, Carnap's use of the Ramsey sentence (as representing the empirical or synthetic content of the original theory using constant theoretical terms) serves a profoundly different purpose than van Fraassen's defense of instrumentalism on the basis of a fundamental epistemological distinction between "acceptance" and "belief." To "accept" a theory, for van Fraassen, is to assert that the observational phenomena are embeddable into some abstract model of the theory-and this, as we pointed out, is precisely what the Ramsey sentence asserts. But to "believe" the theory, in van Fraassen's terms, is to go much further and assert that the theory itself is true- and van Fraassen therefore agrees with the majority of contemporary scientific realists that the truth of the theory is quite distinct from the truth of its Ramsey sentence. For Carnap, by contrast, no such gap between empirical adequacy and truth makes logical (semantical) sense: there is no room remaining for an instrumentalist to "accept" the theory without being simultaneously committed to its truth. This is how, from Carnap's 
point of view, he entirely dissolves the purely philosophical debate between realism and instrumentalism while also preserving the genuinely important insights of both positions. ${ }^{\mathrm{x}}$

Of course Carnap's attempted dissolution, as noted at the beginning, satisfies neither side of the contemporary debate. But this, from Carnap's point of view, is because both sides remain trapped in a fruitless meta-physical — that is, meta-scientificproject: that of giving the "correct" ontology and epistemology for modern science from a distinctively philosophical perspective. And it is precisely here, by contrast, that the true philosophical radicalism of Carnap's own position clearly emerges. At the very beginning of his semantic period Carnap published "Von der Erkenntnistheorie zur Wissenschaftslogik," arguing that all traditional epistemological projects, including his own earlier project in the Aufbau, must now be renounced as "unclear mixture[s] of psychological and logical components" $(1936,36)$. As Carnap also explains, whereas both traditional metaphysics and Kant's synthetic a priori have already been overcome by scientific empiricism, the present (and even more difficult) task is to overcome all forms of traditional epistemology as well-including the earlier epistemology of the Vienna Circle. In particular, what Carnap now calls Wissenschaftslogik is in no way concerned with either explaining or justifying our scientific knowledge by exhibiting its ultimate basis; it is rather concerned with developing a new role for philosophy vis-à-vis the empirical sciences that will maximally contribute to scientific progress while, at the same time, avoiding all the traditional philosophical disputes and obscurities which have constituted (and, according to Carnap, continue to constitute) serious obstacles to progress in both the sciences and scientific philosophy. ${ }^{\mathrm{xi}}$ 
Armed with the new logico-mathematical tools of modern logic, the Carnapian logician of science can participate, together with the scientists themselves, in the articulation, clarification, and development of formal inferential frameworks for articulating empirical theories and testing them by experimental methods. Beginning in the mid to late 1940s, for example, Carnap did just this with respect to the probabilistic and statistical inferential frameworks now being applied with ever-increasing frequency in the physical, biological, and social sciences. However, unlike the empirical scientists themselves (the physicists, biologists, and social scientists), the logician of science, as such, is not concerned with then actually testing empirical theories within such inferential frameworks. Moreover, unlike the applied mathematician (for example, the statistician), who also develops formal methods for use in the empirical sciences, the logician of science has a characteristically philosophical interest in developing a systematic method for dissolving persistent and unresolvable metaphysical controversies which, in Carnap's view, constitute an ever-present obstacle to scientific progress. ${ }^{\text {xii }}$ In the case of his work on the logical foundations of probability and statistical inference, for example, Carnap was especially concerned with dissolving the traditional philosophical debate about the "true nature" of probability—subjective or objective—by drawing a sharp distinction between two different concepts of probability: logical or epistemic (degree of confirmation) and empirical or physical (long-run relative frequency). ${ }^{\text {xii }}$

Carnap's stance vis-à-vis the empirical sciences is therefore quite different from the perspective we are familiar with in either traditional philosophy or most recent philosophy of science. Carnap does not attempt to answer any general questions about the possibility or ultimate justification of scientific knowledge; nor, a fortiori, does he 
attempt to give any general philosophical answer to the question of whether we should take the theoretical entities postulated by empirical science to be "real." Carnap does not attempt, with van Fraassen, to make philosophical room for agnostic instrumentalism, nor, with contemporary scientific realism, to provide some kind of general "explanation" for the success of scientific practice on the basis of the "ontological reality" of theoretical entities. Carnap rather attempts, as we have seen, systematically to dissolve this philosophical dispute using distinctively logical methods. Indeed, it is this last task, in particular, that makes Carnapian Wissenschaftslogik characteristically philosophical and explains why, for Carnap, the formal tools we employ must be drawn from mathematical logic (and not simply from informal mathematics-as employed, for example, by statisticians or mathematical physicists). Whereas formal logic, throughout much of the modern philosophical tradition (beginning with Leibniz and culminating in the twentiethcentury mathematical philosophy articulated by Frege and Russell) has had a fundamental importance for epistemology and metaphysics, mathematical logic, in Carnap's hands, has a fundamental importance for anti-epistemology and antimetaphysics instead: its role is precisely to safeguard our ongoing practice of developing empirical scientific theories within formal mathematical frameworks from epistemological and metaphysical contamination.

Carnap's treatment of the problem of theoretical terms within his version of the axiomatic method therefore has both a positive and a negative dimension. For most of this paper I have concentrated on the negative side: the way in which Carnap's approach liberates us from the intractable philosophical debate between instrumentalism and realism. The only "ontological" question that now matters concerns the existence of an 
appropriate mathematical structure into which the observable phenomena are to be embedded - and this question, in turn, is answered within the ongoing practice of modern mathematical physics itself. For the great advances of modern mathematical physics, from Carnap's point of view, consist precisely in the discovery of appropriate systems of abstract axioms (and correspondence rules) characterizing the mathematical structures in question. Indeed, this fundamental feature of modern physics, as we have seen, provided Carnap with the initial motivations for his distinctive approach to theoretical terms when he first seriously considered this topic in $1939 .{ }^{\text {xiv }}$ Even in 1939, therefore, Carnap was also envisioning a positive purpose for Wissenschaftslogik, in so far as we could then address the axiomatic foundations of modern physics (together with mathematical physicists) entirely free of all metaphysical distractions.

Carnap discusses the axiomatic foundations of quantum mechanics, in particular, in the final chapter of Philosophical Foundations of Physics. It is not yet clear, he says, how the language of physics must change in response to the fundamentally non-classical character of quantum probabilities, but Carnap is sure, nonetheless, that the modern axiomatic method represents our very best hope for progress:

I am convinced that two tendencies, which have led to great improvements in the language of mathematics during the last half century, will prove equally effective in sharpening and clarifying the language of physics: the application of modern logic and set theory, and the adoption of the axiomatic method in its modern form, which presupposes a formalized language system. In present-day physics, in which not only the content of theories but the entire conceptual 
structure of physics is under discussion, both those methods could be of an enormous help.

Here is an exciting challenge, which calls for close cooperation between physicists and logicians—-better still, for the work of younger men who have studied both physics and logic. The application of modern logic and the axiomatic method to physics will, I believe, do much more than just improve communication among physicists and between physicists and other scientists. It will accomplish something of far greater importance: it will make it easier to create new concepts, to formulate fresh assumptions. An enormous amount of new experimental results has been collected in recent years, much of it due to the great improvement of experimental instruments, such as the big atom smashers. On the basis of these results, great progress has been made in the development of quantum mechanics. Unfortunately, efforts to rebuild the theory, in such a way that all the new data fit into it, have not been successful. Some surprising puzzles and bewildering quandaries have appeared. Their solution is an urgent, but most difficult task. It seems a fair assumption that the use of new conceptual tools could here be of essential help. $(1966 / 74,291)^{\mathrm{xv}}$

The very last paragraph of both the chapter and the book (already quoted before) immediately follows these words. ${ }^{\text {xvi }}$ We can now appreciate the full import, I hope, of Carnap's sincere and deeply felt—but nevertheless metaphysically neutral—commitment to structuralism. ${ }^{\text {xii }}$ 


\section{Notes}

${ }^{i}$ See Psillos (2001, 153-154). This paper includes Carnap's previously unpublished lecture, "Theoretical Concepts in Science," delivered in Santa Barbara on December 29, 1959.

ii As Martin Gardiner explains in his Forward to the second edition (1974, v-vi): "In response to a friendly letter from Grover Maxwell, Carnap agreed (shortly before his death in 1970) that his all-too-brief comments on the conflict between instrumentalism and realism, with respect to the nature of scientific theory, be clarified. With this in mind, he made certain alterations on the two pages [255-256], and added a new footnote referring to a 1950 paper which gives his views in more detail."

iii For discussion see Demopoulos and Friedman (1985). The problem has recently been rigorously generalized by Demopoulos $(2003,2007)$ to apply directly to Carnap's use of the Ramsey sentence.

iv This point is first emphasized in Demopoulos (2003).

${ }^{\mathrm{v}}$ For the case of quantum mechanics, in particular, see Carnap (ibid., 210-211): "If we demand from the modern physicist an answer to the question what he means by the symbol ' $y$ ' of his calculus, and are astonished that he cannot give an answer, we ought to realize that the situation was already essentially the same in classical physics. There the physicist could not tell us what he meant by the symbol ' $E$ ' in Maxwell's equations. An "intuitive understanding" or a direct translation of ' $E$ ' into terms referring to observable properties is neither necessary nor possible. The situation of the modern physicist is not essentially different. He knows how to use the symbol ' $y$ ' in the calculus in order to derive predictions which we can test by observations. (If they have the form of 
probability statements, they are tested by statistical results of observations.) Thus the physicist, although he cannot give us a translation into everyday language, understands the symbol ' $y$ ' and the laws of quantum mechanics. He possesses that kind of understanding which alone is essential in the field of knowledge and science."

${ }^{v i}$ For discussion of Hempel's treatment of the Ramsey sentence and the Craig reaxiomatization see Friedman (2008). The present paper extends and further develops my discussion of Carnap there.

vii Thus, I cannot follow Demopoulos $(2003,2007)$ in considering (Demopoulos's generalization) of the Newman problem to be a fatal objection to Carnap's view of theoretical terms. In particular, Demopoulos criticizes Carnap's use of the Ramseysentence for requiring only the existence of an appropriate mathematical structurewhich, as Demopoulos shows, is "almost analytic" in so far as it logically follows from the totality of the observational consequences of $\mathrm{T}$ together with a cardinality assumption. Carnap's own view, however, is that the synthetic content of T does not exceed its empirical content, and he aims to defend this view, moreover, against the metaphysical excesses of both "realism" and "instrumentalism." Demopoulos, from this point of view, is relying on a fundamentally "realist" intuition about what the (synthetic) content of a scientific theory should be taken to be.

viii Carnap makes this clear in Philosophical Foundations of Physics (1966/74, 253-254: "Ramsey certainly did not mean—and no one has suggested—that physicists should abandon theoretical terms in their speech and writing. To do so would require enormously complicated statements. For example, it is easy to say in the customary [realistic] language that a certain object has a mass of five grams.... [But] the translation 
of even this brief sentence into the Ramsey language demands an immensely long sentence, which contains the formulas corresponding to all the theoretical postulates, all the correspondence postulates, and their existential quantifiers. . . . It is evident that it would be inconvenient to substitute the Ramsey way of speaking for the ordinary discourse of physics in which theoretical terms are used[, so that] physicists find it vastly more convenient to talk in the shorthand language that includes theoretical terms, such as 'proton', 'electron', and 'neutron'."

${ }^{\text {ix }}$ From this point of view, the theoretical constants are really term-forming operators rather than genuine non-logical constants, and it is precisely this that is then made completely explicit in the e-operator variant. For details see Carnap (1961), Psillos (2000).

${ }^{x}$ See especially the conclusion of the chapter on the Ramsey sentence in the first edition of Philosophical Foundations of Physics (1966, 256): "It is obvious that there is a difference between the meanings of the instrumentalist and the realist ways of speaking. My own view, which I shall not elaborate here, is that the conflict between the two approaches is essentially linguistic. It is a question of which way of speaking is to be preferred under a given set of circumstances. To say that a theory is a reliable instrument-that is, that the predictions of observable events that it yields will be confirmed-is essentially the same as saying that the theory is true and that the theoretical, unobservable entities it speaks about exist. Thus, there is no incompatibility between the thesis of the instrumentalist and that of the realist. At least, there is no incompatibility so long as the former avoids such negative assertions as, '. . . but the theory does not consist of sentences which are either true or false, and the atoms, 
electrons, and the like do not really exist'." Van Fraassen's agnosticism, by contrast, needs to make room for precisely the assertion that the theory is empirically adequate but its postulated theoretical entities do not exist.

xi Carnap already anticipated the replacement of traditional philosophy by Wissenschaftslogik in The Logical Syntax of Language in 1934 (§72 ): "Wissenschaftslogik takes the place of the inextricable tangle of problems known as philosophy.'

xii Of course the claim that "unresolvable" metaphysical controversies always constitute an obstacle to scientific progress is itself controversial. I myself think that many such controversies in the history of science-e.g., the controversy between Leibniz and Clarke at the beginning of the eighteenth century-have in fact been scientifically quite fruitful. What Carnap himself has especially in mind is the "crisis" in the foundations of mathematics of the late 1920s and early 1930s, where logicism, formalism, and intuitionism opposed one another in what might be reasonably to be taken to be a less than entirely fruitful manner.

xiii See Zabell (2007) for discussion of how Carnap's work on the logical foundations of statistical inference, although somewhat outside of the mainstream, did in fact interact fruitfully with that of working statisticians.

${ }^{\text {xiv }}$ Compare the paragraph to which note $\mathrm{v}$ above is appended.

${ }^{\mathrm{xv}}$ As we saw in note $\mathrm{v}$ above, the case of quantum mechanics was an especially important part of Carnap's motivations for originally proposing the partial interpretation view of theoretical terms in 1939. To see what conceptual problems Carnap might have in mind here compare Carnap's earlier discussion of the importance of the analytic/synthetic 
distinction in modern physics (1966/74, 257-258): "In my opinion, a sharp analytic/synthetic distinction is of supreme importance for the philosophy of science. The theory of relativity, for example, could not have been developed if Einstein had not realized that the structure of physical space and time cannot be determined without physical tests. He saw clearly the sharp dividing line that must always be kept in mind between pure mathematics, with its many types of logically consistent geometries, and physics, in which only experiment and observation can determine which geometries can be applied most usefully to the physical world. This distinction between analytic truth (which includes logical and mathematical truth) and factual truth is equally important today in quantum theory, as physicists explore the nature of elementary particles and search for a field theory that will bind quantum mechanics to relativity."

${ }^{\text {xvi }}$ For convenience I reproduce it again (1966/74, 291-292): "Some physicists believe that there is a good chance for a new breakthrough [in our understanding of quantum mechanics] in the near future. Whether it will be soon or later, we may trust—provided the world's leading statesman refrain from the ultimate folly of nuclear war and permit humanity to survive-that science will continue to make great progress and lead us to ever deeper insights into the structure of the world."

xvii Carnap's metaphysically neutral commitment to structuralism is similar to that developed by Howard Stein (1989)— —which is by no means surprising as Stein had been a student of Carnap's at the University of Chicago. Nevertheless, while Stein gives primarily historical arguments for his position, Carnap's stance (as is typical with him) is primarily logical. Stein (1989) was delivered at the same conference where John Worrall (1989) first initiated contemporary structural realism. 


\section{References}

Carnap, R. (1934). Logische Syntax der Sprache. (Wien: Springer); translated as The logical syntax of language. (London: Kegan Paul, 1937)

Carnap, R. (1936). Von der Erkenntnistheorie zur Wissenschaftslogik. (In Actes du Congrès international de philosophie scientifique, fasc. 1: Philosophie scientifique et empirisme logique (pp. 36-41). Paris: Hermann.)

Carnap, R. (1939). Foundations of logic and mathematics. (In O. Neurath, R. Carnap, \& C. Morris (Eds.). International encyclopedia of unified science, vol. I (pp. 139-213). Chicago: University of Chicago Press.)

Carnap, R. (1950). Empiricism, semantics, and ontology. Revue Internationale de Philosophie, 11, 20-40.

Carnap, R. (1956). The methodological character of theoretical concepts. (In H. Feigl \& M. Scriven (Eds.). Minnesota studies in the philosophy of science, vol. I: The foundations of science and the concepts of psychology and psychoanalysis (pp. 38-76). Minneapolis: University of Minnesota Press.)

Carnap, R. (1958). Beobachtungssprache und Theoretische Sprache. Dialectica, 12, 236248.

Carnap, R. (1961). On the use of Hilbert's e-operator in scientific theories. (In Y. BarHillel et al. (Eds.) Essays on the foundations of mathematics (pp. 156-164). Jerusalem: The Magnus Press.)

Carnap, R. (1963). Replies and systematic expositions. (In Schilpp (1963, 859-1013).) 
Carnap, R. (1966). Philosophical foundations of physics: An introduction to the philosophy of science. (New York: Basic Books)

Carnap, R. (1974). An introduction to the philosophy of science. (New York: Basic Books)

Demopoulos, W. \& Friedman, M. (1985). Critical notice: Bertrand Russell's The Analysis of Matter: Its historical context and contemporary interest. Philosophy of Science, 52, 621-639.

Demopoulos, W. (2003). On the rational reconstruction of our theoretical knowledge. British Journal for the Philosophy of Science, 54, 371-403.

Demopoulos, W. (2007). Carnap on the rational reconstruction of scientific theories. (In Friedman \& Creath (2007).)

Friedman, M. (2008). Wissenschaftslogik: The role of logic in the philosophy of science. Synthese, 164, 385-400.

Friedman, M. \& Creath, R. (Eds.) (2007). The Cambridge companion to Carnap. (Cambridge: Cambridge University Press)

Hempel, C. G. (1958). The theoretician's dilemma: A study in the logic of theory construction. (In H. Feigl, M. Scriven, \& G. Maxwell (Eds.). Minnesota studies in the philosophy of science, vol. II: Concepts, theories and the mind-body problem (pp. 37-98). Minneapolis: University of Minnesota Press.)

Hempel, C. G. (1963). Implications of Carnap's work for the philosophy of science. (In Schilpp (1963, 685-709).)

Newman, M. H. A. (1928). Mr. Russell's causal theory of perception. Mind, 37, 137-148. 
Psillos, S. (1999). Scientific realism: how science tracks truth. (London \& New York: Routledge)

Psillos, S. (Ed.) (2000). Rudolf Carnap's 'Theoretical Concepts in Science'. Studies in History and Philosophy of Science, 31, 151-172.

Schilpp, P. (Ed.) (1963). The philosophy of Rudolf Carnap. (La Salle: Open Court)

Van Fraassen, B. C. (1980). The scientific image. (Oxford: Clarendon Press)

Stein, H. (1989). Yes, but . . . some skeptical remarks on realism and anti-realism. Dialectica, 43, 47-65.

Worrall, J. (1989). Structural realism: the best of both worlds? Dialectica, 43, 99-124.

Zabell, S. L. (2007). Carnap on probability and induction. (In Friedman \& Creath (2007).) 